\title{
Instantaneous Optimal Control of Seismic Response using Magnetorheological Damping
}

\author{
N. K. Chandiramani and S. P. Purohit \\ Department of Civil Engineering, Indian Institute of Technology Bombay, Powai, Mumbai, 400076, India
}

(Received 19 May 2011, accepted 13 October 2011)

Instantaneous Optimal Control (IOC) is used to obtain the desired force from an MR damper fitted to a seismically excited building. Excitation is considered when minimizing performance index, unlike in classical optimal controllers. The modified Bouc-Wen damper model and on-off voltage law is considered. Various forms of state weighting matrices are considered for controller design. IOC is compared with Linear Quadratic Regulator (LQR), Linear Quadratic Gaussian (LQG), and Passive-on control. IOC yields reduction in maximum peak and maximum RMS interstorey drift and in accelerations vis-à-vis LQR and LQG. The IOC Riccati Matrix Type controller appears most effective, yielding the lowest maximum peak drift, the lowest maximum peak acceleration, and the best storeywise drift control when compared to passive-on, LQR, and LQG. The controller also yields substantially lower peak accelerations when compared to LQR and LQG.

\section{INTRODUCTION}

Semiactive devices are useful for vibration control since their properties can be adjusted in real time and since they have relatively low power requirements. Magnetorheological (MR) dampers are semiactive devices, using MR fluids having controllable yield characteristics, which produce a sizeable damping force for small input voltage. Their hysteritic behavior can be represented using the modified Bouc-Wen model having voltage dependent parameters. ${ }^{1}$ Dominguez et al. developed a current-frequency-amplitude dependent BoucWen model for MR dampers. ${ }^{2}$ Other contributions include the phase-transition model of Wang and Kamath, ${ }^{3}$ and the modified LuGre friction model of Jiménez and Àlvarez-Icaza ${ }^{4}$ and Sakai et al. ${ }^{5}$

Various methods for controller design have been used with MR dampers. Due to the difficulty in inverting damper dynamics, predicting applied voltage that produces a desired damper force becomes a challenging task. Hence, various voltage laws have been proposed. Dyke et al. used acceleration-feedbackbased Linear Quadratic Gaussian (LQG) control, with the modified Bouc-Wen model and an on-off Clipped Voltage Law based on desired and measured damper forces. ${ }^{1}$ Chang and Zhou used Linear Quadratic Regulator (LQR) control with a recurrent neural network model for damper inverse dynamics. ${ }^{6}$ Yuen et al. used reliability-based robust linear control with a clipped voltage law. ${ }^{7}$ Prabakar et al. used $H_{\infty}$ control and genetic searching to enhance the performance of the semiactive system. $^{8}$

Yang et al. proposed instantaneous optimal control (IOC) algorithms by including seismic excitation when minimizing the quadratic performance index (PI) at each instant. ${ }^{9}$ Results showed that IOC is somewhat more efficient than classical LQR control, and IOC is easy to implement. Subsequently, using Lyapunov's direct method, Yang et al. determined various structures of state weighting $\mathbf{Q}$ that yield stable and effective controllers. ${ }^{10}$ Agrawal and Yang presented an optimal con- troller (non-IOC) using a PI that was quadratic in control input and polynomial in states, the minimization of which led to a polynomial control law and Riccati and Lyapunov equations for the gains. ${ }^{11}$ The controller used less control energy than LQR control. Ribakov and Dancygier ${ }^{12}$ compared IOC with velocity and acceleration feedback ${ }^{10}$ and polynomial control ${ }^{11}$ in an implementation with a controlled stiffness damper. Renzi and Serino considered a structural bracing system with MR dampers operating in passive and semi-active on-off mode. ${ }^{13}$ An on-off control algorithm was derived by instantaneously minimizing a time-dependent quadratic PI, comprising states but not control input. Chang and Yang derived an instantaneous optimal control law based on Newmark integration of the second-order equations of motion, with weighting matrices selected to yield unconditionally stable control, and they applied the same to an active tendon system. ${ }^{14}$

Among the few available applications of IOC for seismic response control, noted above, none exist for a system with MR dampers with the PI containing the damper force. Further, comparison of IOC with other well-established controllers for MR damper applications, such as LQG, are not available. The goals of this paper are to:

(i) implement IOC (with full-state feedback and velocity and acceleration feedback) for a seismically excited system with MR dampers, for various weighting matrices yielding stable control; and

(ii) assess the performance amongst the various ensuing IOC controllers, and also compare these with passive-on, LQR, and LQG control (that neglect the excitation during PI minimization).

Thus, the IOC controller due to Yang et al..$^{9,10}$ is considered for a MR damper fitted to a building. The modified BoucWen damper model and a Clipped Voltage $\mathrm{Law}^{1}$ is used to obtain the damper voltage required to produce a desired control force. Various available structures of state weighting $(\mathbf{Q})$ that 